\title{
Prediction of steel plate deformation due to triangle heating using the inherent strain method
}

\author{
Chang Doo Jang ${ }^{1}$, Tae Hoon Kim², Dae Eun $\mathrm{Ko}^{3}$, Thomas Lamb ${ }^{4}$, and Yun Sok Ha \\ ${ }^{1}$ Department of Naval Architecture and Ocean Engineering, Seoul National University, San 56-1, Sillim dong, Kwanak-gu, Seoul 151-744, \\ Korea \\ ${ }^{2}$ LG EDS, Korea \\ ${ }^{3}$ Samsung Heavy Industries, Korea \\ ${ }^{4}$ University of Michigan, USA \\ ${ }^{5}$ Seoul National University, RIMSE, Korea
}

\begin{abstract}
In a shipyard, line heating and triangle heating are two major processes carried out by skilled workers to form curved plates in various shapes under various heating conditions. There have been many studies on line heating, but triangle heating has rarely been studied owing to its complicated heating process with irregular multiheating paths and highly concentrated heat input. Triangle heating is the most labor-intensive job. Hence, it is essential for most shipyards to study the automation, as well as the improvement, of the triangle heating process in order to increase hull-forming productivity. In this study, a pioneering attempt to simulate triangle heating was made. A circular disk-spring model is proposed as an analysis model for the elastoplastic procedure of triangle heating, and the inherent strain method is also used to analyze the deformation of plates. The results of the simulation were compared with those of experiments and showed good agreement. It is shown that the present approach and the model used in this study are effective and efficient for simulating triangle heating for the steel plate forming process in shipbuilding.
\end{abstract}

Key words Triangle heating - Thermal elastoplastic analysis · Inherent strain region

\section{List of symbols}

$b$ breadth of the inherent strain region for the elliptical type

$b_{z \text { e }}$ breadth of the inherent strain region according to $z$ for the elliptical type

$b_{z \mathrm{t}}$ breadth of the inherent strain region according to $z$ for the trapezoid type

$b_{1}$ the longer breadth of the inherent strain region for the trapezoid type

$b_{2}$ the shorter breadth of the inherent strain region for the trapezoid type

$d$ maximum depth of the inherent strain region for the elliptical type

Address correspondence to: C.D. Jang (cdjang@snu.ac.kr) Received: September 10, 2004 / Accepted: June 17, 2005 $h$ depth of the inherent strain region for the trapezoid type $\varepsilon^{*} \quad$ inherent strain

\section{Introduction}

In a shipyard, line heating and triangle heating are two major processes carried out by skilled workers to form curved plates in various shapes under various heating conditions. The heating process is selected according to the shape of the curved plate required. The line heating process is used to form plates of the saddle type or the twisted type, whereas the triangle heating process is mainly applied to the concave type. Many studies have been carried out on line heating, but triangle heating has rarely been studied owing to its complicated heating process, with irregular multiheating pathways and highly concentrated heat input.

Triangle heating has mainly been used to form the bow and stern plates of ships' hulls, which is the most labor-consuming job (Fig. 1). Hence, it is essential for shipyards to develop a more efficient fabrication system for the automation, as well as the improvement, of triangle heating processes in order to increase productivity.

In this study, a pioneering attempt to simulate triangle heating was made. An analysis model of triangle heating using the inherent strain method ${ }^{1}$ is proposed, and the computation procedure for the size and shape of the inherent strain distribution is described. The inherent strain is the sum of elastically irrecoverable strains which induce permanent deformation of the plate. For example, plastic strain, thermal strain, and phase transformation strain are typical inherent strains. To induce inherent strain, a circular-disk spring model (Fig. 2) was proposed for 2D thermal elastoplastic analysis. The spring model is a simulation of the elastic restraint from the surrounding area outside the disk 
against expansion or shrinkage of the disk due to temperature changes. The results of the analysis were compared with the experimental results to validate the usefulness and effectiveness of the proposed method.

\section{Simplified analysis model for triangle heating}

In this study, a simplified analysis model of triangle heating is proposed, as shown in Fig. 3. When triangle heating is carried out, the breadth and depth of the inherent strain region are raised from the starting point (C) to the end point $\left(\mathrm{C}^{\prime}\right)$ of the heating process. In this model, it is assumed that the inherent strain region consists of line-heating elements, each of which have increased breadth and depth in the $x$-direction. Because the breadth and depth of each element increases linearly, they are taken as increasing linearly from the first element to the last one in order to simplify the computations. ${ }^{2}$ Based on the results of heat transfer analysis and experiments, it was found that the inherent strain region goes through the thickness of the plate at the end edge, as shown in Fig. 3d. In this case, the shape of the inherent strain region is a trapezoid.

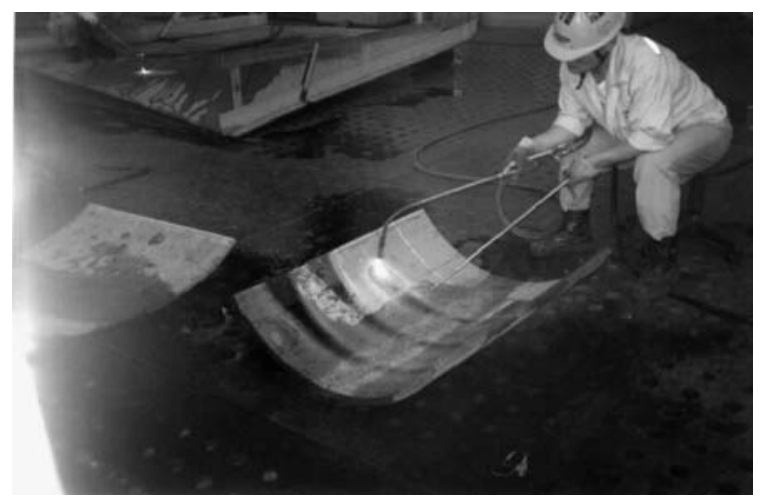

Fig. 1. Triangle heating in a shipyard

\section{Determination of the inherent strain region}

The inherent strain region is assumed to be a region where the maximum temperature exceeds Ac1 $\left(\approx 700^{\circ} \mathrm{C}\right) \cdot{ }^{3}$ From the experimental results, it was found that the section pertaining to the inherent strain region is as shown in Fig. $3 c$ and d. In the section at $A-A^{\prime}$, the shape of the inherent strain region is elliptical, and the equation for the breadth-depth relation is as given in Eq. 1. In the section at $\mathrm{B}-\mathrm{B}^{\prime}$, the shape of the inherent strain region is a trapezoid, and the equation for the breadth-depth relation is as given in Eq. 2. These two shapes are determined by whether the depth of heat penetration is more than the thickness of the plate or not. From the experimental results, the reason why the isotemperature line changes from a curve to a straight line after full penetration is that the conduction speed along the back surface is much faster than the heat convection speed to the air.

$$
\begin{aligned}
& b_{z \mathrm{e}}=b \sqrt{1-\frac{1}{d^{2}}\left(z-\frac{h}{2}\right)^{2}} \\
& b_{\mathrm{zt}}=\frac{b_{1}-b_{2}}{h} z+\frac{b_{1}+b_{2}}{2}
\end{aligned}
$$

\section{Computation of equivalent loads for finite-element analysis}

Equivalent loads can be calculated by integrating the inherent strains in the cross section. After applying these equivalent loads to the plate, the final thermal deformation due to triangle heating can be obtained through elastic structural analysis. By integrating the inherent strains, the shrinkage force per unit length and the bending moment per unit length can be calculated, as in Eqs. 3 and 4.1,4

$$
f=\int_{\frac{h}{2}}^{\frac{h}{2}-d} \frac{b_{z}}{b} E \varepsilon^{*} \mathrm{~d} z
$$
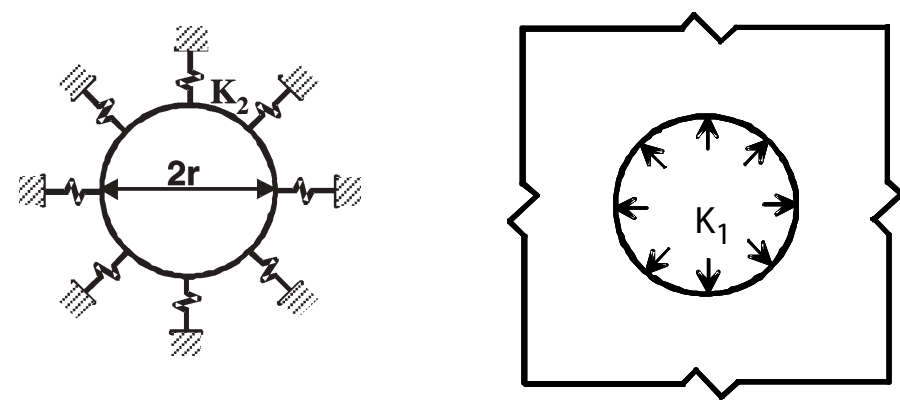

Fig. 2. Circular-disk spring model 


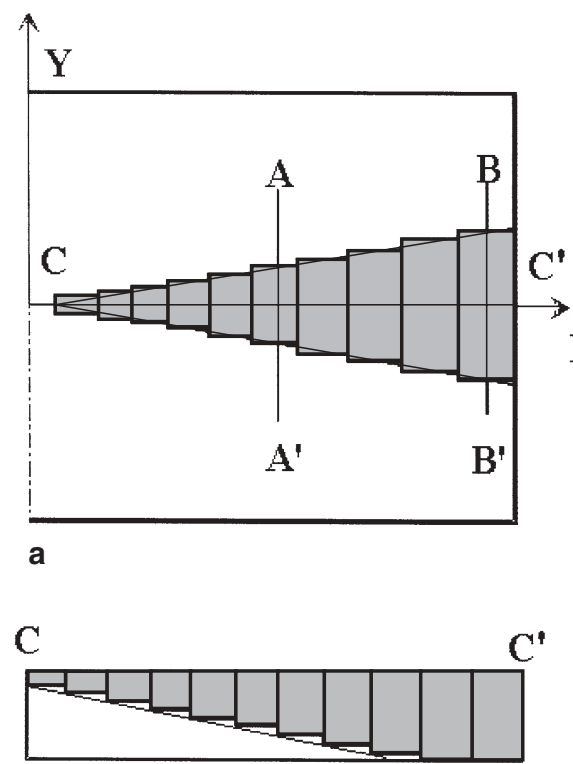

b
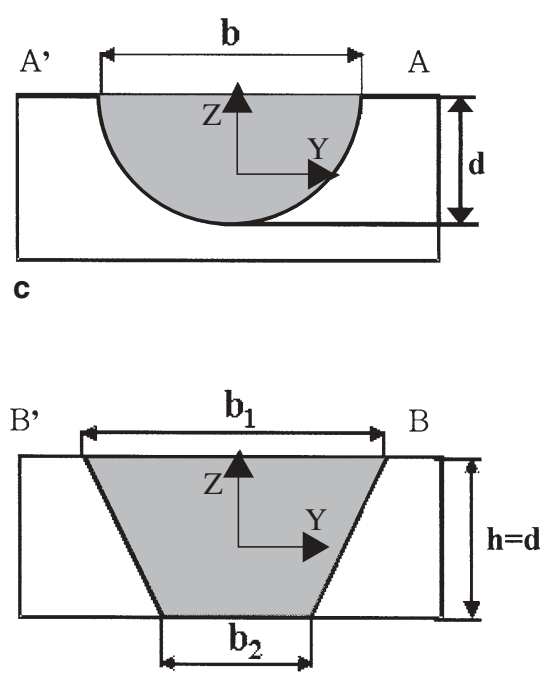

d
Fig. 3. Analysis model for triangle heating. a plan view. $\mathbf{b} \mathrm{C}-\mathrm{C}^{\prime}$ section. c $\mathrm{A}-\mathrm{A}^{\prime}$ section. $\mathrm{d} \mathrm{B}-\mathrm{B}^{\prime}$ section

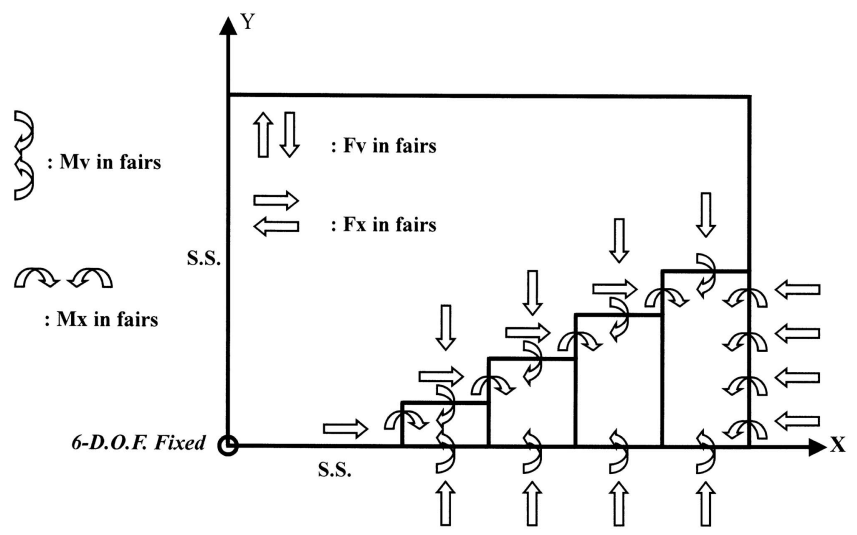

Fig. 4. Equivalent loads in the analysis model

$m=\int_{\frac{h}{2}}^{\frac{h}{2}-d} \frac{b_{z}}{b} E \varepsilon^{*} z \mathrm{~d} z$

The distribution of the shrinkage forces and bending moments is illustrated in Fig. 4. The test models are supported in a suitable way to prevent deflections due to the weight of the steel plates.

\section{Deformation of a flat plate}

Figure 5 shows the specifications of the specimen plate, and Table 1 gives the conditions chosen for the triangle heating experiment. ${ }^{5}$ The triangle heating was performed on a flat plate $20-30 \mathrm{~mm}$ thick, as shown in Fig. 5. Experimental results on the longitudinal deflection

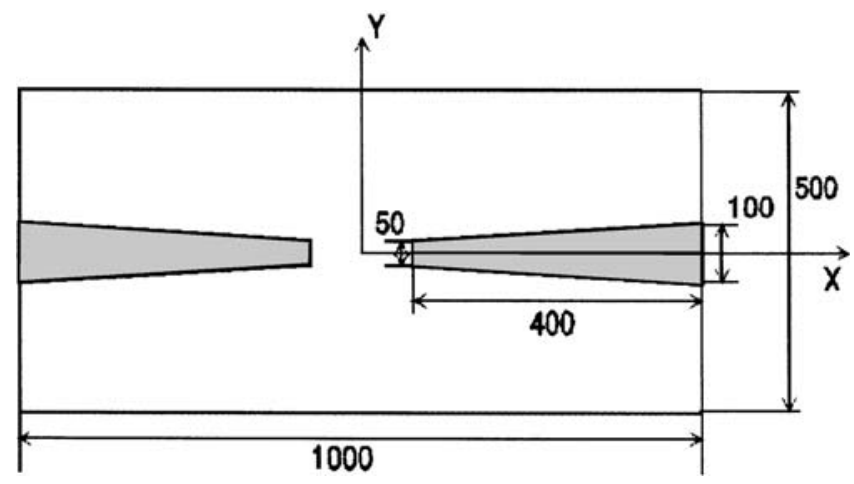

Fig. 5. Specifications of plate specimen (mm)

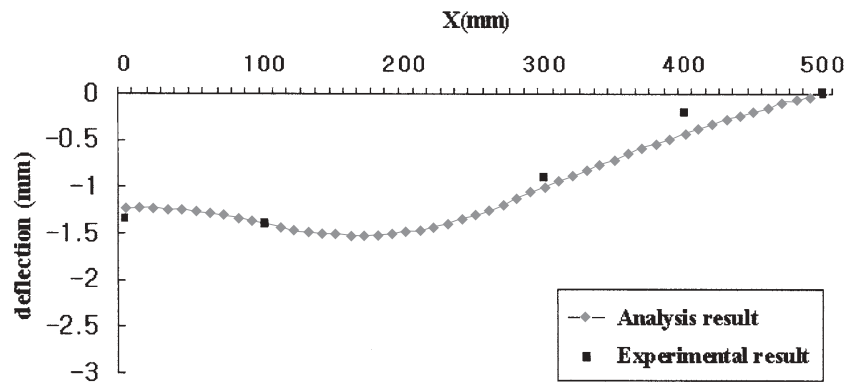

Fig. 6. Longitudinal deflection of a $30-\mathrm{mm}$ plate at $y=0 \mathrm{~mm}$

and transverse shrinkage were obtained and were compared with the analysis results. ${ }^{6}$

Figures 6 and 7 show the longitudinal deflections of the $30-\mathrm{mm}$ plate at $y=0 \mathrm{~mm}$, and at $y=250 \mathrm{~mm}$. In Fig. 6 , the analysis results are almost the same as the experimental results at $x=0-100$, but a difference appears in 
Table 1. Conditions for the triangle heating experiment for a flat plate

\begin{tabular}{lcll}
\hline Thickness & Heating velocity & \multicolumn{1}{c}{ Heating conditions } & \multicolumn{1}{c}{ Material } \\
\hline $20 \mathrm{~mm}$ & $130 \mathrm{~mm} / \mathrm{min}$ & Oxygen pressure $700 \mathrm{KPa}$ & A-grade mild steel \\
$25 \mathrm{~mm}$ & $100 \mathrm{~mm} / \mathrm{min}$ & Propane pressure $70 \mathrm{KPa}$ & \\
$30 \mathrm{~mm}$ & $80 \mathrm{~mm} / \mathrm{min}$ & Water cooling & \\
\hline
\end{tabular}

Table 2. Conditions for the triangle heating experiment for a cylindrical plate

\begin{tabular}{lcccc}
\hline Thickness & Heating velocity & Radius of curvature & Heating conditions & Material \\
\hline $30 \mathrm{~mm}$ & $120 \mathrm{~mm} / \mathrm{min}$ & $1000 \mathrm{~mm}$ & $\begin{array}{l}\text { Oxygen pressure 700 Kpa } \\
\text { Propane pressure 70 KPa } \\
\text { Water cooling }\end{array}$ & A-grade mild steel \\
& & & Wat \\
\hline
\end{tabular}

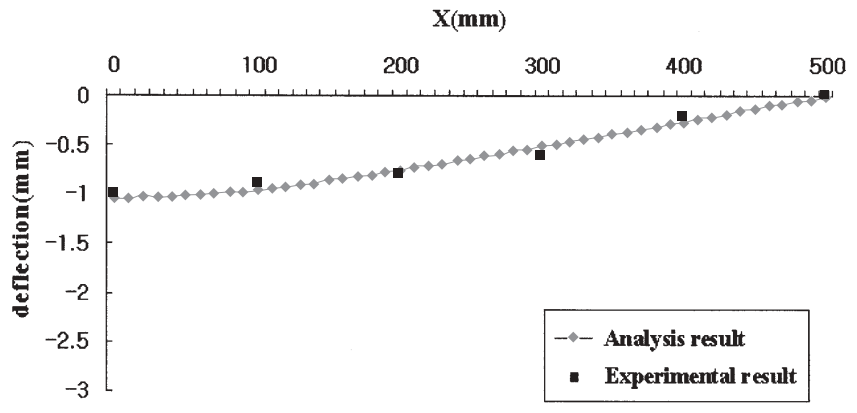

Fig. 7. Longitudinal deflection of a $30-\mathrm{mm}$ plate at $y=250 \mathrm{~mm}$

the latter part of the heating process. In the analysis, the FEM commercial code (ANSYS 9.0) was used. The elements of the model were selected by shell type, and the mesh size was almost square $(1 \mathrm{~cm} \times 1 \mathrm{~cm})$.

\section{Comparison of analysis results and experimental results}

When the plate is heated the heated surface becomes lumpy. The deformations caused by this phenomenon cannot be calculated numerically. Therefore, the difference observed in the latter part of the heating process $(x=400 \mathrm{~mm})$ is caused by this physical phenomenon and the phase-transformation effect. The inherent strain in this method does not contain phase transformation strain because the water-cooling temperature is assumed to be sufficiently low. However, the value and tendency of the deflections in the analysis results are in good agreement with those in the experimental results, as shown in Figs. 6 and 7.

Figure 8 shows the analysis and experimental results for transverse shrinkages, when triangle heating is performed on a flat plate $30 \mathrm{~mm}$ thick. The analysis results show a fairly good agreement with the experimental

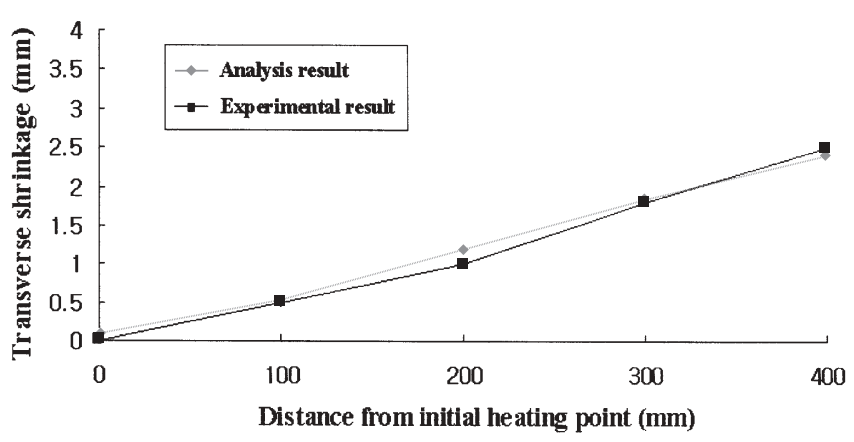

Fig. 8. Transverse shrinkage of a $30-\mathrm{mm}$ plate

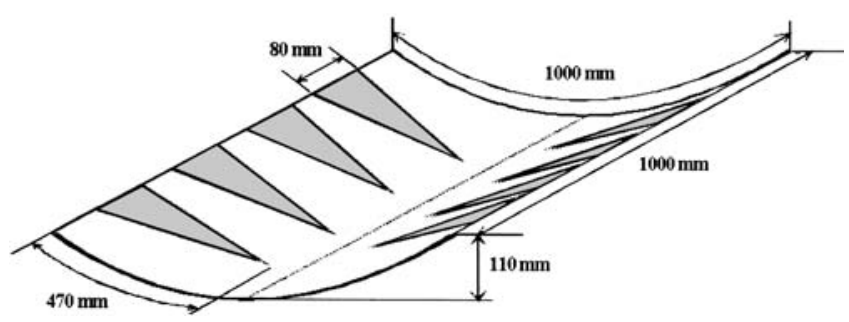

Fig. 9. Specifications of a cylindrical plate

results. Thus, the proposed simplified analysis model for triangle heating is validated.

\section{Deformation of a cylindrical plate}

In this section, the proposed and validated triangleheating model is used for an analysis of the deformations of a cylindrical plate used for manufacturing concave curved plates, and the analysis results are compared with experimental results. Table 2 shows the experimental conditions, and Fig. 9 shows the specifications of a cylindrical plate. A quarter-cylinder plate model was used in the analysis. Figure 10 shows the deformation of a quarter-cylinder plate. As shown in 


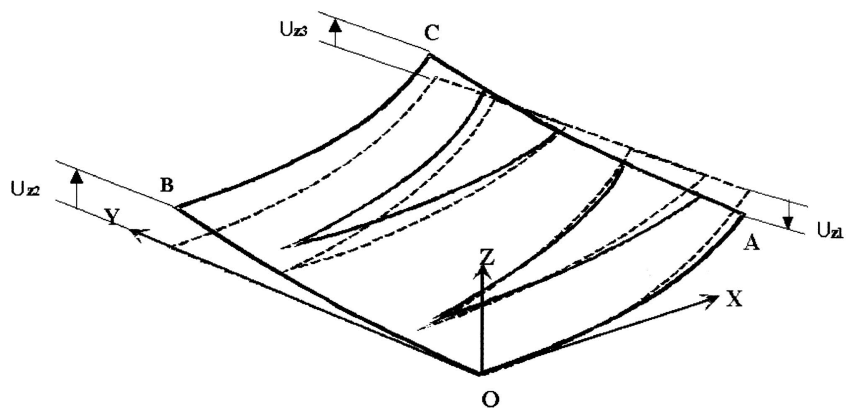

Fig. 10. Deformation of a cylindrical plate

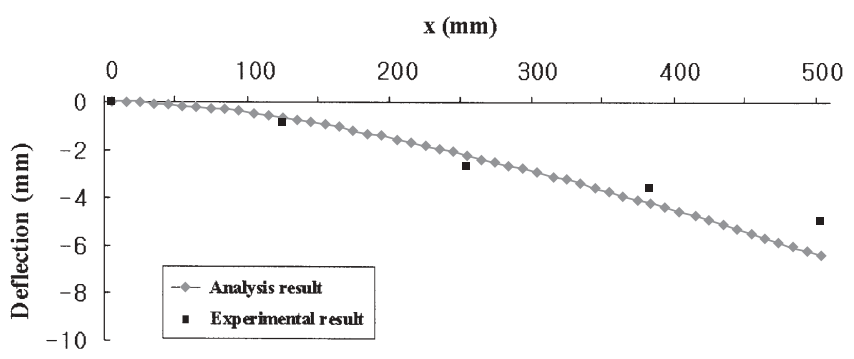

Fig. 11. Longitudinal deflection of a cylindrical plate

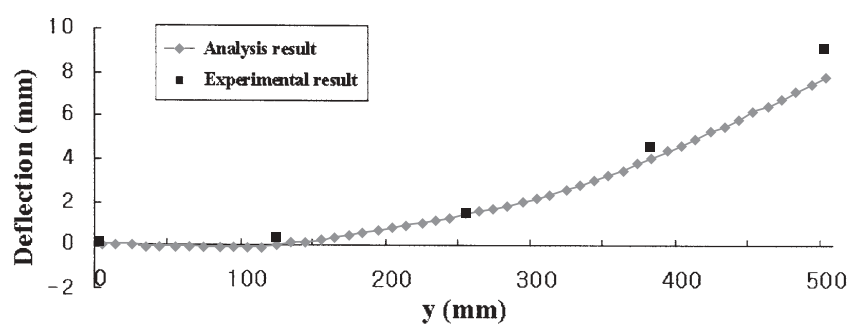

Fig. 12. Transverse deflection of a cylindrical plate

Fig. 10, point $\mathrm{A}$ is deflected downward from the origin, and points $\mathrm{B}$ and $\mathrm{C}$ are deflected upward from the origin. The three-dimensional deformations can be observed, but in this analysis only longitudinal deflections $(\mathrm{O}-\mathrm{A}, \mathrm{B}-\mathrm{C})$ and transverse deflections $(\mathrm{O}-\mathrm{B}, \mathrm{A}-\mathrm{C})$ were considered. In other words, the original value and the deflection of points $\mathrm{A}, \mathrm{B}$, and $\mathrm{C}$ were compared in the $z$-direction. Figure 11 shows the longitudinal deflections in the analysis result, and the experimental result at $y=0(\mathrm{O}-\mathrm{A}$ in Fig. 10). Figure 12 shows the transverse deflections in the analysis result, and the experimental result at $x=0$ (O-B in Fig. 10).

The results of this simulation were compared with those from experiments and showed good agreement. The analysis results for the cylindrical plate using the analysis model of triangle heating proposed well correspond to the experimental results in terms of value and tendency. Thus, the approach and model used in this study are verified to be an effective and efficient to simulate triangle heating for the steel plate forming process in shipbuilding.

\section{Conclusions}

The main conclusions from this study are summarized below.

1. A pioneering attempt to simulate the steel plate forming process by triangle heating was successfully accomplished.

2. From the results of the heat transfer analysis and the cutting test, it was confirmed that the shape of the zone affected by heat exceeding $\mathrm{Ac} 1\left(\approx 700^{\circ} \mathrm{C}\right)$ is elliptical or trapezoidal according to the plate thickness and the heating conditions.

3. The circular disk-spring model is acceptable as an analysis model for the elasto-plastic procedure of triangle heating.

4. A simplified approach to predicting deformations of a steel plate due to triangle heating was proposed based on inherent strain analysis, and the results of this simulation showed good agreement with those of experiments with flat and cylindrical plates.

5. This approach and the model used in this study were shown to be an effective and efficient to simulate triangle heating for the steel plate forming process in shipbuilding

6. The complicate thermal elasto-plastic problem of triangle heating was formulated into a simple elastic analysis with high computation efficiency. Therefore, this approach can be applied to real-time control of the triangle heating process.

7. This approach based on inherent strain analysis provided a basis for developing an improved and automated plate-forming system by triangle heating.

Acknowledgments. This work was supported by a Korea Research Foundation grant (KRF-2002-013E00028). The authors express sincere gratitude for the support. The authors also thank and Hyun Soo Ryu, Seoul National University, for his help in preparing this manuscript.

\section{References}

1. Jang CD, Seo SI, Ko DE (1997) A study on the prediction of deformations of plates due to line heating using a simplified thermal elasto-plastic analysis. J Ship Prod 13:1

2. Shin JG, Moshaiov A (1991) Modified strip model for analyzing the line heating method. Part 1. Elastic plates. J Ship Res 35:2 
3. Jang CD, Ha YS, Ko DE, et al (2002) Determination of inherent strain region for plate bending analysis due to line heating. J Soc Nav Archit Korea 39:1

4. Nomoto T, Ohmori T, Satoh T, et al (1990) Development of simulator for plate bending by line heating. J Soc Nav Archit Jpn 168

5. Watanabe M, Satoh K (1961) Effect of welding conditions on the shrinkage distortion in welded structures. Welding J 40
6. Seo SI, Jang CD (1999) A study on the prediction of deformations of welded ship structures. J Ship Prod 15:2

7. Jang CD, Seo SI, Yang YH (2001) Development of a new finite element analysis of deformation of plate due to line heating. J Ship Prod 17:1

8. Ueda Y, Yamakawa T (1973) Analysis of thermal elastic-plastic behavior of metals during welding by finite element method. J Jpn Welding Soc 42:6 